\begin{tabular}{|l|l|l||}
\hline \multicolumn{2}{|c|}{ PublisherInfo } \\
\hline \hline PublisherName & $:$ & BioMed Central \\
\hline \hline PublisherLocation & $:$ & London \\
\hline \hline PublisherImprintName & $:$ & BioMed Central \\
\hline \hline
\end{tabular}

\title{
Using chloroplasts to produce drugs
}

\begin{tabular}{|l|l|l||}
\hline \multicolumn{2}{|c|}{ ArticleInfo } \\
\hline \hline ArticleID & $:$ & 3626 \\
\hline \hline ArticleDOI & $:$ & $10.1186 /$ gb-2000-1-2-reports0049 \\
\hline \hline ArticleCitationID & $:$ & reports0049 \\
\hline \hline ArticleSequenceNumber & $:$ & 23 \\
\hline \hline ArticleCategory & $:$ & Paper report \\
\hline ArticleFirstPage & $:$ & 1 \\
\hline \hline ArticleLastPage & $:$ & 4 \\
\hline \hline & & RegistrationDate : 2000-4-30 \\
ArticleHistory & $:$ & Received \\
\hline \hline ArticleCopyright & $:$ & BioMed Central Ltd2000-4-30 \\
\hline \hline ArticleGrants & $:$ & \\
\hline \hline
\end{tabular}




\begin{tabular}{|l|l|l||}
\hline ArticleContext & $:$ & 130591122 \\
\hline
\end{tabular}

\section{Todd Richmond}

\section{Abstract}

Large quantities of biologically active human somatotropin have been successfully produced in plants, highlighting the promise of plant biotechnology for producing pharmaceuticals.

\section{Significance and context}

One of the most potentially useful applications of plant biotechnology is the production of xenogenic (foreign) proteins. The ability to scale-up production easily by planting additional acreage and the avoidance of disease contaminants from animal and bacterial systems are two of the most attractive potential benefits. Previous work in the field has concentrated on vaccine and antibody production, and on the synthesis of industrial enzymes. Staub et al. now demonstrate the in planta production of biologically active human somatotropin $(\mathrm{hST})$, the primary use for which in medicine is the treatment of hypopituitary dwarfism in children.

\section{Key results}

The production of xenogenic proteins in plants requires a number of optimization steps: choosing the right promoter, finding the correct codon usage, targeting the protein to the proper subcellular compartment, blocking potential degradation, and ensuring proper post-translational modification. Problems with any of these steps can lead to little or no protein production, or to synthesis of a biologically inactive protein. Staub et al. had to overcome several of these problems. Initial expression attempts using traditional transgenesis into the nuclear genome resulted in little or no hST production, so the authors instead used transformation of chloroplasts. Transformation of the plastid genome has several advantages. First, a plant cell can contain up to 10,000 plastid genomes, greatly increasing the possibility of high expression of the transgene. Staub et al. report that hST expression in one of the transgenic lines made up 7\% of total soluble protein. Second, plastid transformation is via homologous recombination. The hST construct was cloned into a vector containing a selectable marker and portions of the plastid genome. This enabled target- specific insertion of the transgene between the $\operatorname{trn} V$ gene and the rps 7/3'-rps 12operon in the plastid genome. This ensures that there are no positional effects for different constructs. Finally, the plastid genome is not transmitted through pollen, allowing greater biological containment of transgenic crops. 
Another difficulty that the authors had to face was how to produce recombinant hST identical to native hST, which contains an amino-terminal phenylalanine residue instead of the typical methionine. When producing therapeutic proteins, the recombinant proteins must be identical to previously characterized proteins, in order to avoid additional clinical trials and re-certification. To recreate the correct amino terminus, normally produced in mammalian systems by cleavage of a signal peptide, the authors created a ubiquitin-hST fusion protein. Cleavage of the protein by ubiquitin protease resulted in the formation of $\mathrm{hST}$ protein with an amino-terminal phenylalanine.

The processed hST was purified from plant extracts, and shown to have the correct disulfide bonds for biological activity: prior to these experiments, it was not clear whether chloroplasts had the molecular machinery for regulated disulfide bond formation. Biological activity was demonstrated using rat lymphoma cell lines that proliferate in the presence of hST. In summary, Staub et al. have demonstrated the feasibility of producing large quantities of an important human therapeutic protein in transgenic plants.

\section{Reporter's comments}

While Staub et al. have successfully demonstrated 'proof-of-concept', there are still technical hurdles to overcome. Although small amounts of correctly processed hST are present in extracts, the major hST protein species contains an amino-terminal proline residue $(\mathrm{P}-\mathrm{hST})$, not a phenylalanine residue $(\mathrm{F}-$ $\mathrm{hST}$ ), as a result of either ubiquitin-processing steps or a secondary protease activity. Although P-hST is biologically active, it is not identical to the protein previously characterized in human clinical trials and presumably would have to be re-evaluated before being used for treatment. Another technical hurdle is the economics of hST purification from plants. Many attempts to produce recombinant proteins in plants have concentrated on deposition of the desired protein in seeds. In this way, existing technology can be used for harvesting and processing, with a minimum of adaptation. In addition, seeds are a natural storage organ, and thus can be used to store the protein for later processing. In this case, the authors will have to develop a purification scheme for extracting hST from fresh vegetative tissue that can be easily scaled-up and that yields very pure protein suitable for pharmaceutical use.

A final issue is whether or not the approach outlined in this article will be generally applicable to other proteins. One major disadvantage of chloroplast protein production is the inability to carry out most post-translational modifications: hST is somewhat unusual in that it undergoes no posttranslational modifications besides signal peptide cleavage and disulfide bond formation. Unlike most mammalian secretory proteins, hST is not glycosylated. Before using this approach more generally, the effect of missing protein modifications on biological activity will have to be evaluated.

\section{Table of links}

Nature Biotechnology 


\section{References}

1. Staub JM, Garcia B, Graves J, Hajdukiewicz PT, Hunter P, Nehra N, Paradkar V, Schlittler M, Carroll JA, Spatola L, et al: High yield production of a human therapeutic protein in tobacco

chloroplasts. Nat Biotechnol. 2000, 18: 333-338. 1087-0156

This PDF file was created after publication. 